\title{
Parâmetros Populacionais do Rebanho Gir Mocho Registrado no Brasil
}

\section{Fábio José Carvalho Faria ${ }^{1}$, Anibal Eugênio Vercesi Filho1, Fernando Enrique Madalena², Luiz Antônio Josahkian ${ }^{3}$}

\begin{abstract}
RESUMO - O objetivo deste trabalho foi descrever a estrutura da população do rebanho Gir Mocho registrado no Brasil. Foram geradas estatísticas descritivas da distribuição do número de progênies, estimados o intervalo de gerações e o tamanho efetivo da população, usando o registro genealógico de animais nascidos entre 1976-1998. Os intervalos de gerações médios estimados para os períodos (1979-1983, 1984-1988, 1989-1993 e 1994-1998) foram, respectivamente, 4,5; 6,05; 7,17 e 9,14 anos. Os tamanhos efetivos da população nos quatro períodos foram, respectivamente, 3, 22, 63 e 70.
\end{abstract}

Palavras-chave: estrutura populacional, intervalo de gerações, tamanho efetivo, variância de progênie

\section{Population Parameters of the Registered Polled Gir in Brazil}

\begin{abstract}
The aim of this study was to describe the population structure of the registered Polled Gir cattle in Brazil. Descriptive statistics of the distribution of the number of progeny, the generation interval and the effective population size were estimated using pedigree records of animals registered from 1976 to 1998. The observed generation interval in each of four periods (1979-1983, 19841988, 1989-1993 and 1994-1998) was, respectively, 4.5, 6.05, 7.17 and $9.14 \mathrm{yr}$. The effective population size for the periods were, respectively, 3, 22, 63 and 70 .
\end{abstract}

Key Words: population structure, generation interval, effective size, progeny variance

\section{Introdução}

Dos principais grupamentos zebuínos trazidos para o Brasil, e que posteriormente tiveram seu registro genealógico, destaca-se a raça Gir, que foi durante algum tempo a raça zebuína com maior número de animais e a mais valorizada. Foi introduzida provavelmente por volta de 1906, e corresponde à raça homônima na Índia (SANTIAGO, 1986), que no seu país de origem é muito utilizada para produção de leite. No início de sua exploração no Brasil era bastante utilizada para corte. Nas regiões tropicais o gado crioulo (Caracú, Crioulo, Curraleiro e outros) foi sendo substituído por animais zebuínos que passaram a ter importância fundamental na exploração pecuária nacional.

O rebanho zebuíno brasileiro foi, em sua grande parte, formado através do cruzamento absorvente de reprodutores de origem Bos indicus com o gado nacional, tendo sido atribuída a característica mocha aos taurinos (SANTIAGO, 1986). O aumento do número de animais mochos no rebanho Gir, intensifi- cou-se na década de 70, e em 1976 foram iniciados os registros no livro genealógico.

O objetivo deste trabalho foi descrever o tamanho de família, o tamanho efetivo e o intervalo de gerações na raça Gir Mocho no Brasil, parâmetros de interesse para o melhoramento e a conservação da raça.

\section{Material e Métodos}

Foram utilizados os dados do registro genealógico da raça Gir Mocho, disponíveis sob processamento eletrônico, da Associação Brasileira de Criadores de Zebu (ABCZ). O arquivo de dados consistiu de 29.146 observações entre os anos de 1976 a 1998, referentes à amostragem de 34.164 registros $(\mathrm{ABCZ}$, 2001). As informações referentes a cada animal consistiam de: pai, mãe, sexo, categoria de registro $(\mathrm{PO}=$ puro de origem; $\mathrm{LA}=$ livro aberto $)$, dia, mês e ano de nascimento, criador e proprietário.

Inicialmente foram geradas estatísticas descritivas sobre esta população. O banco de dados foi

\footnotetext{
1 Aluno de Doutorado em Ciência Animal, Departamento de Zootecnia, Escola de Veterinária da UFMG, Av. Antônio Carlos 6627, Cx. P. 567, CEP 30123-970, Belo Horizonte - MG. E-mail: fariafjc@dedalus.Icc.ufmg.br

2 Professor do Departamento de Zootecnia, Escola de Veterinária da UFMG, Av. Antônio Carlos 6627, Cx. P. 567, CEP 30123-970, Belo Horizonte - MG. E.mail: fermadal@dedalus.lcc.ufmg.br

3 Superintendente técnico da Associação Brasileira de Criadores de Zebu, Praça Vicente Rodrigues da Cunha, 110 BI 1, CEP $38022-330$, Uberaba - MG. Professor de Melhoramento Animal da Faculdade de Agronomia e Zootecnia de Uberaba - FAZU.
} 
separado em quatro períodos, compreendendo os anos 1979-1983, 1984-1988, 1989-1993 e 1994-1998. O intervalo médio de gerações $(\bar{L})$ para cada período foi calculado de acordo a seguinte fórmula:

$$
\bar{L}=1 / 4 *\left(\mathrm{~L}_{\mathrm{PM}}+\mathrm{L}_{\mathrm{PF}}+\mathrm{L}_{\mathrm{MM}}+\mathrm{L}_{\mathrm{MF}}\right)
$$

em que: $\mathrm{L}_{\mathrm{PM}}=$ intervalo médio entre pais e filhos; $\mathrm{L}_{\mathrm{PF}}=$ intervalo médio entre pais e filhas; $\mathrm{L}_{\mathrm{MM}}=$ intervalo médio entre mães e filhos; $\mathrm{L}_{\mathrm{MF}}=$ intervalo médio entre mães e filhas.

Estimou-se também o intervalo médio de gerações dos pais e avós do último período como: pai de pai, pai de mãe, mãe de mãe e mãe de pai. Em cada período foram calculados o número de machos e fêmeas em reprodução, a média, variância e covariância do número de progênie (filhos e filhas por reprodutor/matriz). O tamanho efetivo da população (Ne) foi calculado para cada período, segundo fórmula proposta por HILL (1972):

$$
\begin{gathered}
\frac{1}{N e}=\frac{1}{16 M \bar{L}}\left[2+\sigma_{\mathrm{mm}}^{2}+2\left(\frac{M}{F}\right) \operatorname{cov}(\mathrm{mm}, \mathrm{mf})+\left(\frac{M}{F}\right)^{2} \sigma_{\mathrm{mf}}^{2}\right]+\frac{1}{16 F \bar{L}}[2+ \\
\left.\left(\frac{F}{M}\right)^{2} \sigma_{\mathrm{fm}}^{2}+2\left(\frac{F}{M}\right) \operatorname{cov}(\mathrm{fm}, \mathrm{ff})+\sigma_{\mathrm{ff}}^{2}\right]
\end{gathered}
$$

em que: $\mathrm{M}=$ número de pais no período; $\mathrm{F}=$ número de mães no período; $\sigma^{2}{ }_{\mathrm{mm}}=$ variância do número de filhos por pai; $\sigma^{2}{ }_{\mathrm{mf}}=$ variância do número de filhas por pai; $\sigma_{\text {fm }}^{2}=$ variância do número de filhos por mãe; $\sigma_{\text {ff }}^{2}=$ variância do número de filhas por mãe; $\operatorname{cov}(\mathrm{mm}, \mathrm{mf})=$ covariância do número de filhos de ambos os sexos por pai; cov $(\mathrm{fm}, \mathrm{ff})=$ covariância do número de filhos de ambos os sexos por mãe.

\section{Resultados e Discussão}

Na Tabela 1, constam o número de criadores distribuídos em classes conforme o número de animais registrados, separados por sexo, no período de 1994 a 1998. A escolha deste período teve o intuito de retratar o número de criadores efetivos da raça, atualmente. No período, constam da amostra registros de 4103 animais, sendo 1827 machos e 2276 fêmeas. O número de criadores neste período foi baixo ( $\mathrm{n}=128)$, existindo também grande concentração de animais pertencentes a poucos criadores, já que dois criadores registraram $30 \%$ dos animais, e 10 registraram mais da metade $55 \%$.

Do total de 11.703 machos registrados entre 1976 e 1998, 738 animais foram identificados como pais. O número médio de filhos e filhas por pai foi 18,6 e 18,8, respectivamente, sendo a média de crias de ambos os
Tabela 1 - Distribuição de criadores de acordo com o número de animais registrados separados por sexo de 1994 a 1998

Table 1 - Distribution of breeders according to number of registered animals and sex, from 1994 to 1998

\begin{tabular}{lrrr}
\hline & $\begin{array}{c}\text { Machos } \\
\text { Males }\end{array}$ & $\begin{array}{c}\text { Fêmeas } \\
\text { Females }\end{array}$ & $\begin{array}{c}\text { Ambos os sexos } \\
\text { Both sexes }\end{array}$ \\
\hline $\begin{array}{l}\text { Número de animais } \\
\text { Number of animals }\end{array}$ & \multicolumn{3}{c}{$\begin{array}{c}\text { Número de criadores } \\
\text { Number of breeders }\end{array}$} \\
\hline $1-25$ & 88 & 95 & 88 \\
$26-50$ & 7 & 9 & 22 \\
$51-75$ & 2 & 4 & 10 \\
$76-100$ & 2 & - & - \\
$101-200$ & - & 3 & 5 \\
$\geq 201$ & 2 & 2 & 3 \\
Total & 101 & 113 & 128 \\
Total & \multicolumn{3}{c}{} \\
\hline
\end{tabular}

sexos de 31,67 . Entre os reprodutores, $2,2 \%$ animais contribuíram com $39 \%$ das filhas e $2,5 \%$ dos animais foram pais de $42 \%$ dos machos nascidos no período estudado. A idade média do pai quando do registro da primeira cria foi de 4,3 anos.

$\mathrm{Na}$ Tabela 2, estão expostos os números de reprodutores separados por classe de acordo com o sexo da progênie. Verificou-se que do total de 738 reprodutores, $79 \%$ contribuíram com o registro de machos, ao passo que $89 \%$ foram pais de fêmeas, evidenciando seleção mais intensa para o registro de machos.

Foram identificadas 10.663 mães entre 1976 e 1998, que representam $61 \%$ das 17.443 fêmeas

Tabela 2 - Distribuição de reprodutores de acordo com o número de filhos separados por sexo

Table 2 - Distribution of sires according to progeny number and sex

\begin{tabular}{lccc}
\hline & $\begin{array}{c}\text { Filhos } \\
\text { Sons }\end{array}$ & $\begin{array}{c}\text { Filhas } \\
\text { Daughters }\end{array}$ & $\begin{array}{c}\text { Ambos os sexos } \\
\text { Both sexes }\end{array}$ \\
\hline $\begin{array}{l}\text { Número de filhos } \\
\text { Number of sires }\end{array}$ & \multicolumn{3}{c}{ Número de reprodutores } \\
Progeny numbers \\
\hline $1-25$ & 499 & 559 & 551 \\
$26-50$ & 51 & 55 & 100 \\
$51-75$ & 16 & 26 & 33 \\
$76-100$ & 6 & 6 & 14 \\
$101-200$ & 7 & 8 & 25 \\
$\geq 201$ & 7 & 7 & 15 \\
Total & 586 & 661 & 738 \\
\hline
\end{tabular}


registradas. O número médio de filhos e filhas por mãe foi 1,58 e 1,59 respectivamente, sendo a média de crias de ambos os sexos de 2,1. A distribuição do número de filhos por mãe, separados por sexo, pode ser visualizada na Tabela 3. Do total de 10.663 mães, $62 \%$ contribuíram com o registro de machos, ao passo que $70 \%$ das mães contribuíram para o registro de fêmeas, demonstrando assim como nos pais, maior seleção para mães de machos.

A idade média das mães ao registro da primeira e da última cria foi 4,86 e 8,87 anos, respectivamente, o que refletiu uma "vida útil" para fins de registro de 4,01 anos. O intervalo médio entre registros da mesma vaca foi 1,68 anos. Ressalva-se que a idade média ao primeiro registro pode não corresponder à idade ao primeiro parto, assim como o intervalo médio entre registros pode não corresponder ao intervalo médio de partos, pelo fato de algumas crias não terem sido registradas.

Levando-se em conta o número médio de crias registradas de 2,1 e "vida útil" de registro de 4,01 anos, foi estimado que uma vaca produziria em média 0,27 bezerros registrados/ano $((2,1-1) / 4,01)$. Segundo a ABCZ (2001), entre 1995 e 2000, 4.861 animais obtiveram registro de nascimento, resultando numa média de 810 registros/ano. Com a estimativa de $0,27 \mathrm{cria} /$ ano/vaca estimou-se uma população média de $3.000(810 / 0,27)$ vacas registradas em reprodução, que formariam os estratos núcleo e multiplicador da raça. Com base em critérios descritos pela FAO (1994), o rebanho Gir Mocho registrado no Brasil encontra-se na categoria de populações "em perigo" (entre 1.000 e 5.000 vacas).

$\mathrm{Na}$ Tabela 4, estão apresentadas as estimativas dos intervalos de gerações para as quatro passagens gaméticas de cada período analisado.
Tabela 3 - Distribuição de matrizes de acordo com o número de filhos separados por sexo

Table 3 - Distribution of dam according to progeny number and sex

\begin{tabular}{lrcc}
\hline & $\begin{array}{c}\text { Filhos } \\
\text { Sons }\end{array}$ & $\begin{array}{c}\text { Filhas } \\
\text { Daughters }\end{array}$ & $\begin{array}{c}\text { Ambos os sexos } \\
\text { Both sexes }\end{array}$ \\
\hline $\begin{array}{l}\text { Número de filhos } \\
\text { Number of sires }\end{array}$ & \multicolumn{3}{c}{$\begin{array}{c}\text { Número de mães } \\
\text { Number of dams }\end{array}$} \\
\hline $1-2$ & 5766 & 6453 & 7715 \\
$3-4$ & 780 & 907 & 2.004 \\
$5-6$ & 130 & 141 & 677 \\
$7-8$ & 11 & 19 & 214 \\
$\geq 9$ & - & 1 & 53 \\
Total & 6687 & 7521 & 10.663 \\
\hline
\end{tabular}

Nota-se o reduzido intervalo obtido no primeiro período, que pode ser atribuído à forma de truncamento dos dados, visto que foram incluídos animais com nascimento a partir do início do registro em 1976. O intervalo de gerações médio obtido entre 1976 a 1998 foi de 7,18 anos. Ao considerar as passagens dos reprodutores com mais de 250 filhos (possivelmente utilizados em inseminação artificial), os intervalos nas passagens pai-filho e pai-filha foram 9,43 e 9,46 anos, respectivamente. Parece que esta estimativa ainda não apresenta estabilidade, pois, os intervalos apresentaram crescimento a cada período, notadamente nos passos pai - filho e pai - filha.

Esses intervalos são similares aos demais encontrados para raças zebuínas criadas no Brasil, e foram descritos por FARIA et al. (2001). Vale salientar o reduzido intervalo de gerações descrito por RAZOOK et al. (1993) nos rebanhos da Estação Experimental de

Tabela 4 - Intervalo de gerações das quatro passagens gaméticas e intervalo médio Table 4 - Generation interval of four gametic pathways and average interval

\begin{tabular}{|c|c|c|c|c|c|}
\hline & \multicolumn{5}{|c|}{$\begin{array}{c}\text { Passagens gaméticas } \\
\text { Gametic pathways }\end{array}$} \\
\hline & $\begin{array}{l}\text { Pai - filho } \\
\text { Sire - son }\end{array}$ & $\begin{array}{c}\text { Pai - filha } \\
\text { Sire - daughter }\end{array}$ & $\begin{array}{c}\text { Mãe - filho } \\
\text { Dam - son }\end{array}$ & $\begin{array}{c}\text { Mãe - filha } \\
\text { Dam - daughter }\end{array}$ & $\begin{array}{l}\text { Média } \\
\text { Average }\end{array}$ \\
\hline $\begin{array}{l}\text { Períodos } \\
\text { Periods }\end{array}$ & & & $\begin{array}{l}\text { Ano } \\
\text { Year }\end{array}$ & & \\
\hline 1979 a 1983 & 4,56 & 4,67 & 4,28 & 4,50 & 4,50 \\
\hline 1984 a 1988 & 6,02 & 5,87 & 6,26 & 6,08 & 6,05 \\
\hline 1989 a 1993 & 7,11 & 7,07 & 7,32 & 7,18 & 7,17 \\
\hline 1994 a 1998 & 10,17 & 10,33 & 7,99 & 8,09 & 9,14 \\
\hline
\end{tabular}

Rev. bras. zootec., 30(6S):1984-1988, 2001 
Zootecnia de Sertãozinho - SP, o que torna evidente a possibilidade de redução neste parâmetro possibilitando a sua otimização para auferir maior ganho genético.

O tamanho efetivo estimado para cada período está descrito na Tabela 5, juntamente com alguns fatores que contribuíram nesta estimativa. Estes valores foram calculados utilizando-se os intervalos de gerações descritos na Tabela 4, os quais são muito similares aos previamente descritos por FARIA et al. (2001b) quando utilizaram o intervalo médio de 1976 a 1998. De acordo com WRIGHT (1938), a oscilação do Ne pode ser devida a fatores como flutuações no tamanho populacional, desvios da razão de sexos de 1:1 e a diferenças no sucesso reprodutivo.
A evolução do tamanho efetivo e a variância do tamanho de família de pais podem ser vistas na Figura 1. Ficam evidentes nestes períodos o decréscimo na variância do número de progênie por reprodutor e a redução na relação fêmea:macho, além desses fatores, aumentaram os intervalos de gerações em cada período, todos estes contribuindo para incrementar os valores dos tamanhos efetivos.

O conceito de tamanho efetivo é extremamente importante na descrição de populações e em programas de melhoramento devido às suas relações com endogamia e deriva genética. Com base nos dados apresentados neste trabalho podese estimar o aumento na taxa anual de endogamia

Tabela 5 - Tamanho efetivo, número de reprodutores, média e variância de número de progênies nos quatro períodos Table 5 - Effective size, number of sires, progeny number average and variance for the analyzed periods

\begin{tabular}{|c|c|c|c|c|}
\hline \multirow[b]{2}{*}{$\begin{array}{l}\text { Períodos } \\
\text { Periods }\end{array}$} & \multirow[b]{2}{*}{$\begin{array}{l}\text { Tamanho efetivo } \\
\text { Effective size }\end{array}$} & \multicolumn{2}{|c|}{$\begin{array}{l}\text { Número de progênie por reprodutor } \\
\text { Progeny number per sire }\end{array}$} & \multirow[b]{2}{*}{$\begin{array}{l}\text { Variância } \\
\text { Variance }\end{array}$} \\
\hline & & $\begin{array}{c}\text { Número de reprodutores } \\
\text { Number of sires }\end{array}$ & $\begin{array}{l}\text { Média } \\
\text { Average }\end{array}$ & \\
\hline 1979 a 1983 & 3 & 135 & 26,41 & $14.526,39$ \\
\hline 1984 a 1988 & 22 & 292 & 27,21 & $4.574,36$ \\
\hline 1989 a 1993 & 63 & 380 & 20,66 & $2.358,49$ \\
\hline 1994 a 1998 & 70 & 219 & 16,14 & $1.615,79$ \\
\hline
\end{tabular}

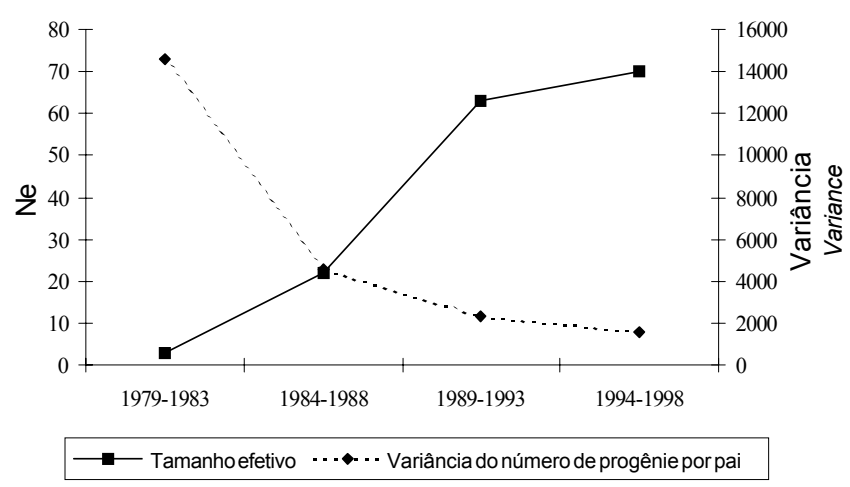

Figura 1 - Tamanho efetivo $\left(\mathrm{N}_{\mathrm{e}}\right)$ e variância do número de progênie por pai na raça Gir Mocho.

Figure 1 - Effective size $\left(N_{e}\right)$ and progeny number variance per sire in the Polled Gir breed. que se apresenta como $\Delta \mathrm{F}_{\mathrm{a}}=1 /\left(2 \mathrm{Ne}^{*}\right)$. Com as devidas substituições, o incremento médio de endogamia estimado ao ano $(1 /(2 \times 70))$ com base no último período foi 0,00714 , o que é relativamente menor que a taxa geralmente admitida de 0,01 ao ano (NICHOLAS, 1980). Recentemente grande enfoque tem sido dado a este parâmetro, pois, conforme GODDARD e SMITH (1990) um Ne mínimo de 40 por geração é necessário para maximizar o retorno econômico em população selecionada para produção de leite, já MEUWISSEN e WOOLLIAMS (1994) recomendam valores entre 30 a 250 para prevenir decréscimo no valor adaptativo. 


\section{Conclusões}

Devido à diminuição do tamanho do rebanho refletido na queda anual de animais registrados, o desenvolvimento da raça Gir Mocho pode ser comprometido futuramente.

A redução da variância do número de progênie, a diminuição da razão fêmea:macho e o aumento no intervalo de gerações contribuíram para o aumento do tamanho efetivo populacional, entretanto, este valor encontra-se no limite mínimo recomendado na literatura para prevenir decréscimo no valor adaptativo.

\section{Agradecimento}

À ABCZ pela concessão dos dados para este trabalho, realizado com apoio do CNPq, CAPES e FAPEMIG.

\section{Referências Bibliográficas}

ABCZ. 2001. "Estatísticas", http://www.abcz.org.br 10/04/2001. FAO. 1994. The management of global animal genetic resources. [Rome, Italy]: FAO, 309 p.

FARIA, F.J.C., VERCESI FILHO, A.E., MADALENA, F.E. et al. 2001. Parâmetros populacionais do rebanho Sindi registrado no Brasil. Rev. bras. zootec., 30(6S):1989-1994.

FARIA, F.J.C., VERCESI FILHO, A.E., MADALENA, F.E. et al. Intervalo de gerações e tamanho efetivo da população da raça Gir Mocho. In: REUNIÃO ANUAL DA SOCIEDADE BRASILEIRA DE ZOOTECNIA, 38, 2001, Piracicaba. Anais... Piracicaba: SBZ, 2001. p.482-483.
GODDARD, M.G., SMITH, C. 1990. Optimum number of bull sires in dairy cattle breeding. J. Dairy Sci., 73:1113-1122.

HILL, W.G. 1972. Effective size of populations with overlapping generations. Theor. Popul. Biol., 3(3):278-288.

MEUWISSEN, T.H.E., WOOLLIAMS, J. 1994. Effective sizes of livestock populations to prevent a decline in fitness. Theor. Appl. Genet., 89:1019-1026.

NICHOLAS, F.W. 1980. Size of population required for artificial selection. Genetical Research, 35:85-115.

RAZOOK, A.G., FIGUEIREDO, L.A., BONILHA NETO, L.M. et al. 1993. Intensidades de seleção e repostas diretas e correlacionadas em 10 anos de progênies de bovinos das raças Nelore e Guzerá selecionadas para peso pós desmame. Bol. Ind. Anim., 50(2):147-163.

SANTIAGO, A.A. 1986. O Zebu na India, no Brasil e no Mundo. Campinas: Instituto Campineiro de Ensino Agrícola, 745 p.

WRIGHT, S. 1938. Size of population and breeding structure in relation to evolution. Science, 87:430-431. 